\title{
Análise de crescimento do milho safrinha em diferentes épocas de aplicação de zinco
}

\author{
Flávio Henrique Ferreira GOMES ${ }^{1 *}$, Fernando Nobre Cunha ${ }^{1}$, Luiz Cesar LOPES FILHO ${ }^{2}$, \\ Frederico Antônio Loureiro SOARES ${ }^{1}$, Marconi Batista TEIXEIRA ${ }^{1}$, Wilker Alves MORAIS \\ ${ }^{1}$ Programa de Pós-Graduação em Ciências Agrárias, Instituto Federal de Educação, Ciência e Tecnologia Goiano, \\ Rio Verde, GO, Brasil. \\ ${ }^{2}$ Programa de Pós-Graduação em Agronomia, Universidade Federal de Goiás, Goiânia, GO, Brasil. \\ *E-mail: gomesfhf@gmail.com
}

Recebido em setembro/2017; Aceito em agosto/2018.

\begin{abstract}
RESUMO: O método de análise de crescimento de plantas é utilizado na avaliação de seu comportamento quando submetidas a práticas agronômicas. Os valores do intervalo de tempo e biomassa permitem a realização de estimativas relacionado ao crescimento de plantas. Objetivou-se, neste trabalho, avaliar a influência das épocas de aplicação de zinco para os índices fisiológicos na cultura do milho safrinha. O delineamento experimental utilizado foi o de blocos ao acaso em esquema de parcelas subdividas $4 \times 5$, com quatro repetições, as parcelas foram compostas por quatro épocas de aplicações (36, 43, 50 e 57 dias após o plantio) e as subparcelas 5 épocas de avaliações $(60,75,90,105$ e 120 dias após o plantio). Os parâmetros da análise de crescimento analisados foram taxa de crescimento da cultura (TCC), taxa de crescimento relativo (TCR), razão de massa foliar (RMF) e taxa de crescimento absoluto (TCA). Para a cultura do milho, a interação entre os fatores Épocas de Aplicação e Épocas de Avaliação (EAp x EAv) foi significativa para todas variáveis analisadas. As épocas de aplicação aos 36 e 43 dias após o plantio, obtiveram os melhores parâmetros de crescimento para cultura do milho safrinha.
\end{abstract}

Palavras-chave: índices fisiológicos, épocas de avaliação, comportamento, massa da matéria seca.

\section{Off-season maize growth analysis in different periods of zinc application}

\begin{abstract}
The plant growth analysis method is used in evaluating its behavior when subjected to agronomic practices. The values of the time interval and biomass allow the realization of estimates related to plant growth. In this work, we aimed at evaluating the influence of zinc-applying periods for physiological indices in the culture of off-season maize. The experimental design used was random blocks in plots subdivided in $4 \times 5$, with four replications, the plots were composed by four periods of applications $(36,43,50$ and 57 days after planting) and subplots by 5 evaluations periods (60, 75, 90, 105 and 120 days after planting). The analyzed growth analysis parameters were culture growth rate (TCC), relative growth rate (TCR), leaf mass ratio (RMF) and absolute growth rate (TCA). For maize culture, the interaction between factors four periods of applications and evaluations periods (EAp x EAv) was significant for all variables analyzed. The periods of application at 36 and 43 days after planting, obtained the best growth parameters for the crop of off-season maize.
\end{abstract}

Keywords: physiological indexes, periods of evaluation, behavior, mass of dry matter.

\section{INTRODUÇÃO}

O milho (Zea mays L.) é cultivado em grande parte do mundo e sua importância econômica é evidenciada pelas diversas formas de utilização, desde a alimentação animal até a indústria de alta tecnologia (OLIVEIRA et. al, 2012).

A consolidação da produção brasileira do milho, reunindo as duas safras (2014/2015), aponta agora para 84.729,2 mil toneladas, representando um acréscimo de $5,8 \%$ em relação à produção passada, que atingiu $80.051,7$ mil toneladas (CONAB, 2015).

Análise de crescimento é um método para a avaliação das diferenças no comportamento de cultivares influenciadas por práticas agronômicas, efeitos de competição ou climáticos, e por fatores intrínsecos associados à fisiologia da planta (ANDRADE et al., 2005; GUIMARÃES et al., 2008). Nesta análise são coletados dados em intervalos de tempos, com os dados de massa seca das partes da planta e da planta toda são realizados diferentes cálculos permitindo realizar uma estimativa do crescimento da planta (SANTOS et al., 2013).

$\mathrm{O}$ acúmulo de matéria seca e o incremento da área foliar, quantificados em função do tempo, são utilizados para estimar vários índices fisiológicos relacionados às diferenças de desempenho entre cultivares ou diferentes materiais da mesma espécie e das comunidades vegetais, nos diversos estudos ecofisiológicos (LIMA et al., 2007). Índices fisiológicos como índice de área foliar, taxas de crescimento da cultura, de crescimento relativo e de assimilação líquida (COSTA et al., 2006; MONTE et al., 2009).

Com a mensuração do crescimento de espécies de interesse agronômico, obtém-se informações que colaboram, consideravelmente, para o aprimoramento das técnicas agrícolas, (OLIVEIRA FILHO et al., 2010). Este trabalho teve por objetivo avaliar a influência das épocas de aplicação de zinco em diferentes épocas de avaliação dos índices fisiológicos para cultura do milho. 


\section{MATERIAL E MÉTODOS}

O experimento foi desenvolvido em condições de campo em sistema de plantio convencional, na estação experimental do Instituto Federal Goiano - Campus Rio Verde (Rio Verde, GO), no ano agrícola 2015. As coordenadas geográficas do local de instalação são $17^{\circ} 48^{\prime} 28^{\prime \prime} \mathrm{S}$ e $50^{\circ} 53^{\prime} 57^{\prime \prime} \mathrm{O}$, com altitude média de $720 \mathrm{~m}$ ao nível do mar. A temperatura média anual varia de 20 a $35^{\circ} \mathrm{C}$ e as precipitações variam de 1.500 a $1.800 \mathrm{~mm}$ anuais e o relevo é suave ondulado $(6 \%$ de declividade). O clima da região é classificado conforme Alvarez et al., (2013), como Aw (tropical), com chuva nos meses de outubro a maio, e com seca nos meses de junho a setembro.

O solo foi classificado como Latossolo Vermelho distroférrico (LVdf), de textura média (SANTOS et al., 2014). As características químicas do solo estão apresentadas na Tabela 1, segundo Donagemma et al. (2011).

$\mathrm{O}$ delineamento experimental utilizado foi o de blocos ao acaso analisado em esquema de parcelas subdividas $4 \times 5$, com quatro repetições, as parcelas foram compostas por quatro épocas de aplicações $(36,43,50$ e 57 dias após o plantio) e as subparcelas 5 épocas de avaliações $(60,75,90,105$ e 120 dias após o plantio).

Foi aplicado um fertilizante mineral simples em suspensão, a base de zinco, via solo, sendo a dose aplicada de $300 \mathrm{ml} \mathrm{ha}^{-1}$. Teor total de Zinco $748 \mathrm{~g} \mathrm{~L}^{-1}$, densidade $1,87 \mathrm{~g}$ $\mathrm{cm}^{-3}\left(25^{\circ} \mathrm{C}\right)$. A semeadura do milho híbrido (P4285YH) foi realizada no dia 06 de março de 2015. Cada parcela foi composta de 8,0 linhas de 4,0 m de comprimento com espaçamento de $0,45 \mathrm{~m}$ entre linhas. Foram eliminadas as duas linhas laterais de cada lado e $1,0 \mathrm{~m}$ de cada extremidade da parcela, avaliando os 2,0 $\mathrm{m}$ de cada uma das 4,0 linhas centrais.

Foram colhidas, em dias pré-determinados, amostras da parte aérea do milho. Após este procedimento, o milho foi transportado e submetido à secagem em estufa de circulação forçada de ar, a $65^{\circ} \mathrm{C}$ até massa constante, foi determinada a massa fresca.

Conforme Benincasa (2003) e Magalhães (1985), após obtido a massa da matéria seca (MS) em intervalos regulares pré-estabelecidos, foram determinados os parâmetros da análise de crescimento. A sequência de cálculos para ser determinados os parâmetros da análise de crescimento em planta foram conforme as equações de 1 a 4 :

$$
\operatorname{TCC}\left(\mathrm{g} \mathrm{m}^{-2} \mathrm{dia}^{-1}\right)=\frac{\left(\mathrm{MS}_{2}-\mathrm{MS}_{1}\right)}{\frac{\mathrm{S}}{\left(\mathrm{t}_{2}-\mathrm{t}_{1}\right)}}
$$

(Equação 1)

em que: Taxa de Crescimento da Cultura (TCC); $\mathrm{S}=$ representa a área amostrada ocupada pela cultura $\left(\mathrm{m}^{-2}\right) ; \mathrm{MS}_{1}$ e $\mathrm{MS}_{2}$, as massas de matéria seca nos tempos inicial $\left(\mathrm{t}_{1}\right)$ e final $\left(\mathrm{t}_{2}\right)$ da avaliação;

$$
\text { TCR }\left(\mathrm{g} \mathrm{g}^{-1} \mathrm{dia}^{-1}\right)=\frac{\left(\operatorname{lnMS}_{2}-\ln \mathrm{MS}_{1}\right)}{\left(\mathrm{t}_{2}-\mathrm{t}_{1}\right)}
$$

em que: Taxa de Crescimento Relativo (TCR); $\ln =$ logaritmo neperiano; $\mathrm{MS}_{1}$ e $\mathrm{MS}_{2}=$ massas de matéria seca nos tempos $\mathrm{t}_{1}$ e $\mathrm{t}_{2}$.

$\operatorname{RMF}\left(\mathrm{g} \mathrm{g}^{-1}\right)=\frac{\mathrm{MS}_{\mathrm{f}}}{\mathrm{MS}_{\mathrm{t}}}$

em que: Razão de Massa Foliar (RMF)MS $\mathrm{M}_{\mathrm{t}}=$ massa de matéria seca total da planta; $\mathrm{MS}_{\mathrm{f}}=$ massa de matéria seca de folha.

$$
\operatorname{TCA}\left(\mathrm{g}^{-1} \mathrm{dia}^{-1}\right)=\frac{\left(\mathrm{MS}_{2}-\mathrm{MS}_{1}\right)}{\left(\mathrm{t}_{2}-\mathrm{t}_{1}\right)}
$$

em que: Taxa de Crescimento Absoluto (TCA) $\mathrm{MS}_{1}$ e $\mathrm{MS}_{2}$ representam a massa da matéria seca nos tempos $t_{1}$ e $t_{2}$.

Os dados obtidos foram submetidos à análise de variância pelo teste $\mathrm{F}$ ao nível de 1 e $5 \%$ de probabilidade, e em casos de significância, foi realizada a análise de regressão à $5 \%$ de probabilidade.

\begin{tabular}{|c|c|c|c|c|c|c|c|c|c|}
\hline & \multicolumn{9}{|c|}{ Características químicas ${ }^{1}$} \\
\hline Profundidade & $\mathrm{N}$ & $\mathrm{P}$ & $\mathrm{S}$ & $\mathrm{Ca}$ & $\mathrm{Mg}$ & $\mathrm{K}$ & $\mathrm{Al}$ & $\mathrm{H}+\mathrm{Al}$ & $\mathrm{pH}$ \\
\hline $\mathrm{m}$ & $\%$ & \multicolumn{2}{|c|}{$---\mathrm{mg} \mathrm{dm}^{-3}---$} & \multicolumn{5}{|c|}{ 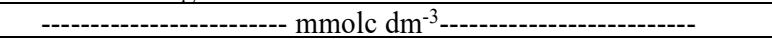 } & $\mathrm{CaCl}_{2}$ \\
\hline $0,00-0,20$ & 0,12 & 4,30 & 2,80 & 2,70 & 0,80 & 63,00 & 0,00 & 3,40 & 5,30 \\
\hline $0,20-0,40$ & 0,07 & 1,80 & 2,00 & 1,70 & 0,50 & 68,00 & 0,00 & 2,30 & 5,40 \\
\hline Profundidade & $\mathrm{Na}$ & $\mathrm{Zn}$ & B & $\mathrm{Cu}$ & $\mathrm{Fe}$ & $\mathrm{Mn}$ & M.O & CTC & $\mathrm{V}$ \\
\hline $\mathrm{m}$ & \multicolumn{6}{|c|}{ 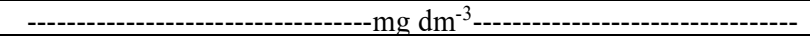 } & $\mathrm{g} \mathrm{dm}^{-3}$ & mmolc dm ${ }^{-3}$ & $\%$ \\
\hline $0,00-0,20$ & 3,0 & 0,6 & 0,17 & 1,8 & 21,5 & 18,3 & 26,0 & 7,07 & 51,95 \\
\hline $0,20-0,40$ & 4,0 & 0,8 & 0,19 & 2,3 & 28,0 & 16,3 & 14,0 & 4,69 & 50,9 \\
\hline
\end{tabular}

Tabela 1. Características químicas do solo da área experimental.

Table 1. Chemical characterization of the soil of the experimental area.

${ }^{1}$ Extrator de P e K, Mehlich ${ }^{-1}$; Matéria Orgânica (M.O); Capacidade de troca de cátions (CTC); Porcentagem de saturação de bases (V).

\section{RESULTADOS}

As épocas de aplicação (EAp) em milho foram significativas $(\mathrm{p}<0,01)$ para todas variáveis analisadas como taxa de crescimento da cultura (TCC), taxa de crescimento relativo (TCR), razão de massa foliar (RMF) e taxa de crescimento absoluto (TCA). Houve efeito significativo ao nível de $1 \%$ de probabilidade, em relação a época de avaliação (EAv) para todas as variáveis analisadas. Para a cultura do milho, a interação entre os fatores (EAp x EAv) foi significante ao nível de $1 \%$ de probabilidade para todas variáveis analisadas (Tabela 2).

Na Figura 1A, no desdobramento dos efeitos dos níveis de época de aplicação, para as épocas de avaliação, é possível constatar que para TCC ocorreu efeito para EAv 60, 75, 90,
105 e 120 dias. Segundo as equações polinomiais obtidas para EAv 60, 75 e 90 dias uma TCC máxima de 26,23; 34,54 e $38,08 \mathrm{~g} \mathrm{~m}^{-2} \mathrm{dia}^{-1}$, respectivamente, foram aproximadamente estimados aos 41 dias após o plantio, para EAv 105 e 120 dias uma TCC máxima de 35,11 e 21,69 $\mathrm{g} \mathrm{m}^{-2} \mathrm{dia}^{-1} \mathrm{~cm}$ foram estimados aos 42 dias após o plantio. Na Figura 1B, no desdobramento dos efeitos das épocas de avaliação, para as épocas de aplicação, é possível constatar que para a TCC houve efeito para EAp aos 36, 43, 50 e 57 dias. Segundo as equações polinomiais obtidas para EAp 36, 43 e 50 dias uma TCC máxima de 37,58; 39,07 e $36,17 \mathrm{~g} \mathrm{~m}^{-2} \mathrm{dia}^{-1}$ foram estimados aos 88 dias após o plantio, para a EAp 57 dias, uma TCC máxima de $34,85 \mathrm{~g} \mathrm{~m}^{-2} \mathrm{dia}^{-1}$ foi estimada aos 87 dias após o plantio. 
$\mathrm{Na}$ Figura 2A, no desdobramento dos efeitos para níveis das épocas de aplicação, para as épocas de avaliação, é possível constatar que para TCR ocorreu efeito para EAv 60, 75 e 90 dias. Segundo as equações polinomiais obtidas para EAv 60, 75 e 90 dias uma TCR máxima de 0,094; 0,056; $0,018 \mathrm{~g} \mathrm{~g}^{-1} \mathrm{dia}^{-1}$, respectivamente, foram estimadas aos 41 dias após o plantio.

Na Figura 2B, no desdobramento dos efeitos das épocas de avaliação, para as épocas de aplicação, é possível constatar que para TCR houve efeito para EAp 36, 43, 50 e 57 dias. Segundo as equações polinomiais obtidas para a EAp 36 dias, uma TCR mínima de $0,006 \mathrm{~g} \mathrm{~g}^{-1} \mathrm{dia}^{-1}$ foi estimada com 111 dias após o plantio, para a EAp 43 dias uma TCR mínima de $0,008 \mathrm{~g} \mathrm{~g}^{-1} \mathrm{dia}^{-1}$ foi estimada com 113 dias após o plantio, para a EAp 50 dias uma TCR mínima de $0,001 \mathrm{~g} \mathrm{~g}^{-1} \mathrm{dia}^{-1}$ foi estimada com 110 dias após o plantio, para a EAp 57 dias uma TCR mínima de $0,001 \mathrm{~g} \mathrm{~g}^{-1} \mathrm{dia}^{-1}$ foi estimada com 107 dias após o plantio.

Na Figura 2A, no desdobramento dos efeitos para níveis das épocas de aplicação, para as épocas de avaliação, é possível constatar que para TCR ocorreu efeito para EAv 60, 75 e 90 dias. Segundo as equações polinomiais obtidas para EAv 60, 75 e 90 dias uma TCR máxima de 0,094; 0,056; $0,018 \mathrm{~g} \mathrm{~g}^{-1} \mathrm{dia}^{-1}$, respectivamente, foram estimadas aos 41 dias após o plantio.
$\mathrm{Na}$ Figura 2B, no desdobramento dos efeitos das épocas de avaliação, para as épocas de aplicação, é possível constatar que para TCR houve efeito para EAp 36, 43, 50 e 57 dias. Segundo as equações polinomiais obtidas para a EAp 36 dias, uma TCR mínima de $0,006 \mathrm{~g} \mathrm{~g}^{-1} \mathrm{dia}^{-1}$ foi estimada com 111 dias após o plantio, para a EAp 43 dias uma TCR mínima de $0,008 \mathrm{~g} \mathrm{~g}^{-1} \mathrm{dia}^{-1}$ foi estimada com 113 dias após o plantio, para a EAp 50 dias uma TCR mínima de $0,001 \mathrm{~g} \mathrm{~g}^{-1} \mathrm{dia}^{-1}$ foi estimada com 110 dias após o plantio, para a EAp 57 dias uma TCR mínima de $0,001 \mathrm{~g} \mathrm{~g}^{-1} \mathrm{dia}^{-1}$ foi estimada com 107 dias após o plantio.

Na Figura 3A, no desdobramento dos efeitos dos níveis das épocas de aplicação, para as épocas de avaliação, é possível constatar que para a RMF ocorreu efeito para EAv 60, 75 e 90 dias. Segundo as equações polinomiais obtidas para EAv 60 dias a RMF máxima de $0,47 \mathrm{~g} \mathrm{~g}^{-1}$ foi estimada aos 40 dias após o plantio, para EAv 75 e 90 dias a RMF máxima de 0,38 e $0,27 \mathrm{~g} \mathrm{~g}^{-1}$ foram estimadas aos 41 dias após o plantio. Na Figura 3B, no desdobramento dos efeitos dos níveis para das épocas de avaliação, para as épocas de aplicação, é possível constatar que para a variável RMF houve efeito para EAp 36, 43, 50 e 57 dias. Segundo as equações polinomiais obtidas para EAp 36, 43, 50 e 57 dias a RMF mínima de 0,$12 ; 0,12 ; 0,13$ e $0,11 \mathrm{~g} \mathrm{~g}^{-1}$ foram estimadas com 120 dias após o plantio.

Tabela 2. Resumo da análise de variância para as variáveis taxa de crescimento da cultura (TCC), taxa de crescimento relativo (TCR), razão de massa foliar (RMF) e taxa de crescimento absoluto (TCA) da cultura do milho submetido a diferentes épocas de aplicação e diferentes épocas de avaliação, em diferentes dias após o plantio.

Table 2. Summary of the variance analysis for the variables culture growth rate (TCC), relative growth rate (TCR), leaf mass ratio (RMF) and absolute growth rate (TCA) of the maize crop submitted to different periods of application and different evaluation periods, on different days after planting.

\begin{tabular}{|c|c|c|c|c|c|}
\hline \multirow{2}{*}{$\mathrm{FV}$} & \multirow{2}{*}{ GL } & \multicolumn{4}{|c|}{ Quadrados Médios ${ }^{1}$} \\
\hline & & TCC & TCR & RMF & TCA \\
\hline EAp & 3,00 & $57,1623 * *$ & $0,0003 * *$ & $0,00719 * *$ & $0,2040 * *$ \\
\hline BLOCO & 3,00 & 0,0397 ns & $0,000007^{\mathrm{ns}}$ & $0,00066^{*}$ & $0,00053^{\mathrm{ns}}$ \\
\hline RESÍDUO (a) & 9,00 & 0,1313 & 0,000018 & 0,00012 & 0,00089 \\
\hline EAv & 4,00 & $740,0189 * *$ & $0,0191 * *$ & $0,2965 * *$ & $8,8314 * *$ \\
\hline EAp * EAv & 12,00 & $0,3546 * *$ & $0,00004 * *$ & $0,00074 * *$ & $0,0105 * *$ \\
\hline RESÍDUO (b) & 48,00 & 0,1268 & 0,000009 & 0,00015 & 0,00124 \\
\hline $\begin{array}{l}\mathrm{CV} \mathrm{a} \\
\mathrm{CV} \mathrm{b}\end{array}$ & & $\begin{array}{l}1,22 \\
1,19\end{array}$ & $\begin{array}{c}12,36 \\
8,99\end{array}$ & $\begin{array}{l}4,04 \\
4,53\end{array}$ & $\begin{array}{l}1,63 \\
1,92\end{array}$ \\
\hline
\end{tabular}

(A)

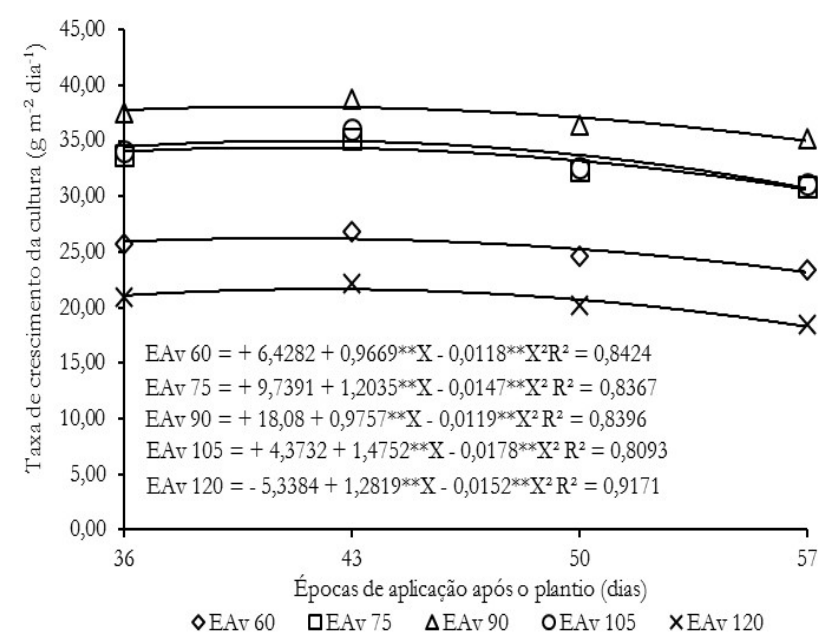

(B)

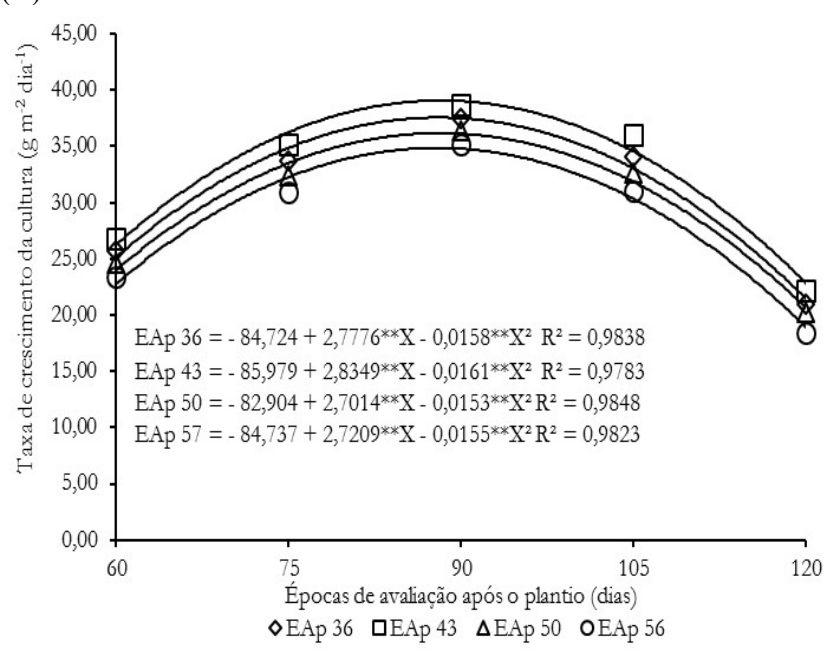

${ }^{* *} \mathrm{e}^{*}$ significativo a 1 e a $5 \%$ de probabilidade pelo teste de $\mathrm{F}$

Figura1. Taxa de crescimento do milho, em função das épocas de aplicação (A) e das épocas de avaliação (B).

Figure 1. Maize growth rate, in function of application periods (A) and evaluation periods (B). 
(A)

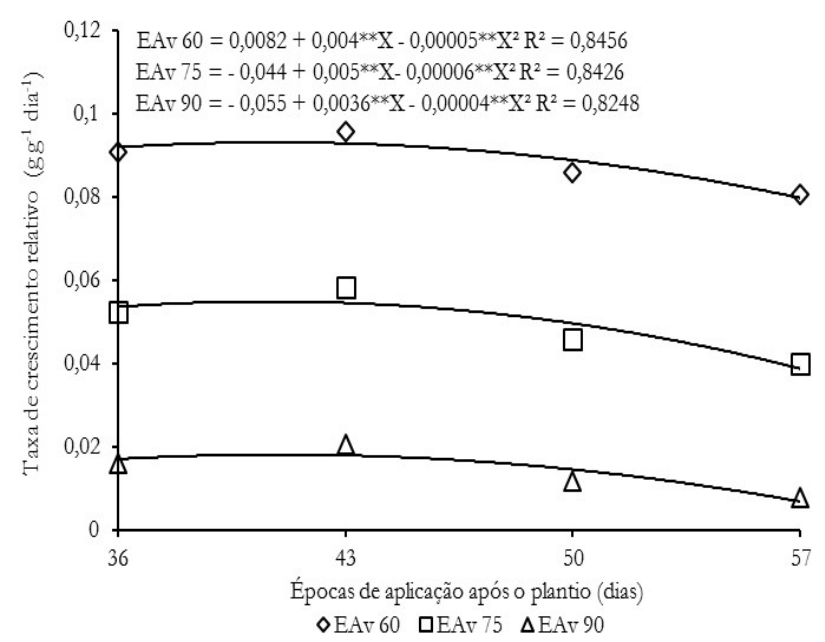

(B)

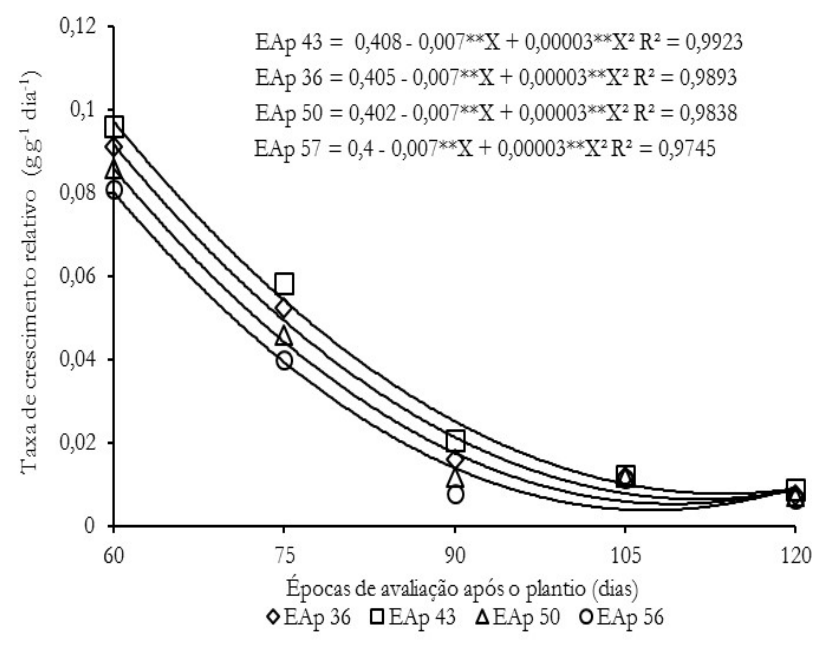

${ }^{* *} \mathrm{e}^{*}$ significativo a 1 e a $5 \%$ de probabilidade pelo teste de $\mathrm{F}$.

Figura2. Taxa de crescimento relativo do milho, em função das épocas de aplicação (A) e das épocas de avaliação (B).

Figure 2. Relative growth rate of maize, in function of application periods (A) and evaluation periods (B).

(A)

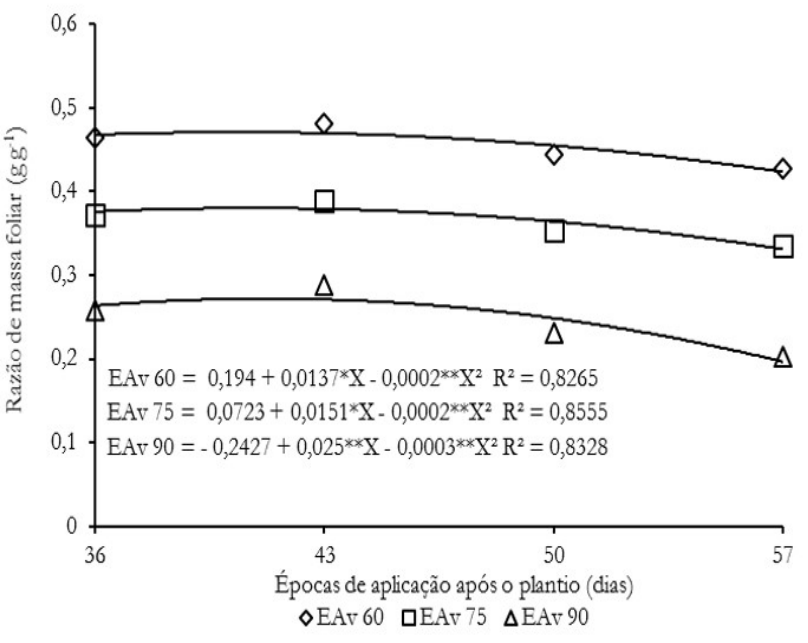

(B)

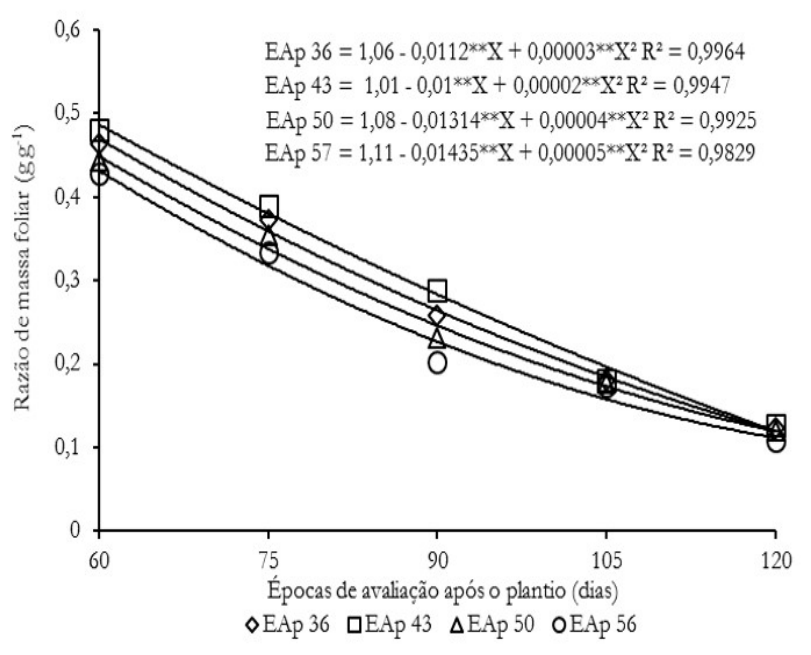

e * significativo a 1 e a $5 \%$ de probabilidade pelo teste de $\mathrm{F}$

Figura3. Razão de massa foliar do milho, em função das épocas de aplicação (A) e das épocas de avaliação (B).

Figure 3. Maize leaf mass ratio, in function of application periods (A) and evaluation periods (B)

Na Figura 4A, e pelo desdobramento dos efeitos dos níveis das épocas de aplicação, para as épocas de avaliação, é possível constatar que para a variável TCA ocorreu efeito para EAv60, 75, 90, 105 e 120 dias. Segundo as equações polinomiais obtidas para EAv 60,75 e 90 dias uma TCA máxima de 3,$04 ; 1,86 ; 2,26 \mathrm{~g} \mathrm{dia}^{-1}$ foram estimadas aos 41dias após o plantio, para EAv 105 dias uma TCA máxima de $1,29 \mathrm{~g} \mathrm{dia}^{-1}$ foi estimada aos 40 dias após o plantio e para EAv 120 dias uma TCA máxima de $1,15 \mathrm{~g} \mathrm{dia}^{-1}$ foi estimada aos 36 dias (épocas de aplicação).

Na Figura 4B, no desdobramento dos efeitos dos níveis das épocas de avaliação, para as épocas de aplicação, é possível constatar que para a TCA houve efeito para EAp 36, 43, 50 e 57 dias. Segundo as equações polinomiais obtidas para EAp 36, 43, 50 e 57 dias uma TCA mínima de 1,15; 1,$14 ; 1,12 ; 1,10 \mathrm{~g} \mathrm{dia}^{-1}$ foi estimada com 120 dias após o plantio.

\section{DISCUSSÃO}

Para a TCC, segundo a equação de regressão quadrática, obteve-se o maior valor na avaliação aos 88 dias quando a aplicação foi realizada aos 41 dias após o plantio. O mesmo comportamento foi observado por Monte et al. (2009) e Alvarez et al. (2012), para cultura do tomate e arroz, respectivamente, onde obteve-se crescimento lento no início, acelerando-se, em seguida, até atingir o valor máximo e reduzindo-se a partir desse ponto, pois à medida que a planta cresce e aumenta o seu número de folhas, ela possui maior capacidade de produzir fitomassa. Em estudo realizado por Lopes (2009) com a cultura do milho, variando sistemas de plantio, observou que em ambos os sistemas de plantio, inicialmente as TCCs foram baixas, posteriormente aumentaram e em seguida os valores foram diminuindo.

Para a TCR, de acordo com a equação de regressão quadrática, obteve-se o maior valor na avaliação aos 60 dias quando a aplicação foi realizada aos 41 dias após o plantio. Júnior Orioli et al. (2008), verificaram que o teor foliar de $\mathrm{Zn}$ foi influenciado pelos diferentes métodos de aplicação, sendo possível o acúmulo do nutriente na massa seca da parte aérea. Oliveira et al. (2005) observaram TCR máxima aos 135 dias após o plantio e, posteriormente, um decréscimo gradual até a colheita. Com o aumento da massa acumulada pela planta, 
ocorre aumento da necessidade de fotoassimilados para a manutenção dos órgãos já formados. Assim, a quantidade de fotoassimilados disponível para o crescimento (TCR) tende a ser menor (BENINCASA, 2003).

Com relação a RMF, segundo a equação de regressão quadrática, foi verificado o maior valor na avaliação aos 60 dias quando a aplicação foi realizada aos 40 dias após o plantio. Prado et al. (2008), constataram que a aplicação de zinco promoveu incremento na altura e na produção de matéria do milho, a aplicação de zinco via solo (incorporado e localizado), foliar e semente foram semelhantes no desenvolvimento inicial da cultura. Em estudo realizado por Lacerda et al. (2010), com as culturas de milho e feijão, observou a redução da RMF no período estudado. Em estudo

(A)

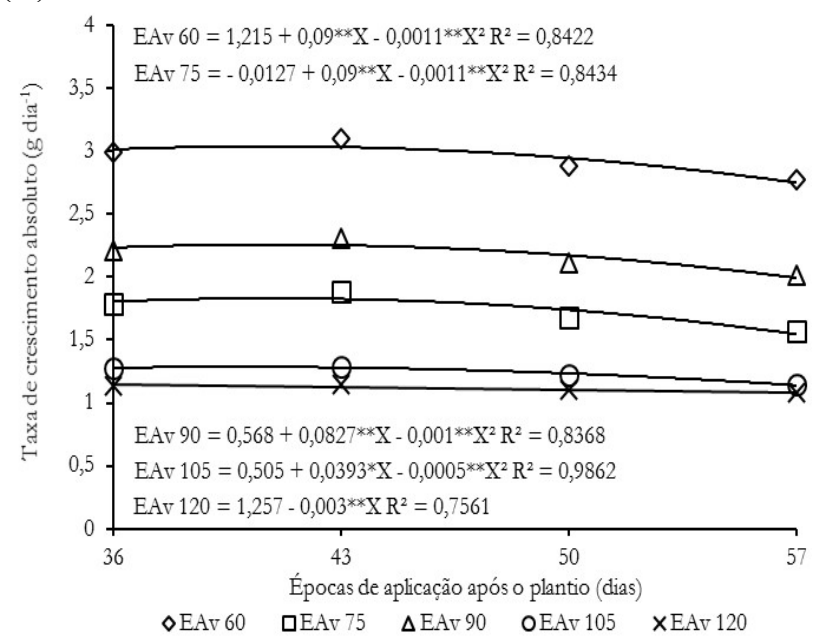

realizado por Falqueto et al. (2009), com duas cultivares de arroz, observou-se o aumento na RMF aos 47 e 50 dias após o plantio, a partir desses períodos, os valores RMF reduziram-se com a ontogenia das plantas. Aumentos em RMF refletem maior alocação de assimilados para as folhas em desenvolvimento, tidas como dreno metabólico preferencial (ZEPKA, 2007).

Segundo a equação de regressão quadrática, verificou-se a máxima TCA na avaliação aos 60 dias com aplicação aos 41 dias após o plantio. Lopes et al. (2011) observaram comportamento quadrático na TCA da cultura do tomate. Andrade et al. (2009), relataram o comportamento da cultura do feijão em relação a TCA que foi decrescendo e chegando a valores mínimos ao fim do ciclo da cultura.

(B)

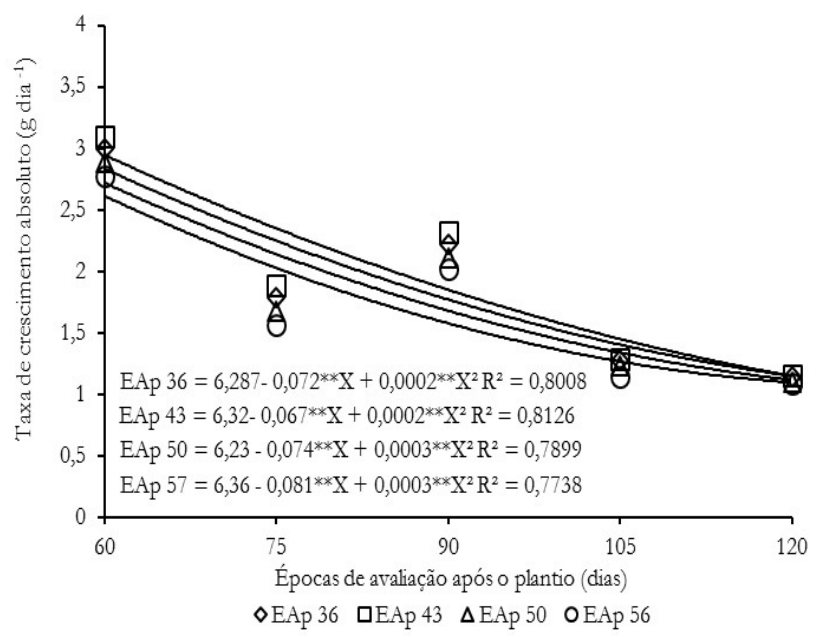

** e ${ }^{*}$ significativo a 1 e a $5 \%$ de probabilidade pelo teste de $\mathrm{F}$.

Figura 4. Taxa de crescimento absoluto do milho, em função das épocas de aplicação (A) e das épocas de avaliação (B).

Figure 4. Absolute growth rate of maize, in function of application periods (A) and evaluation periods (B).

\section{CONCLUSÕES}

Os resultados obtidos, mostraram que a época de aplicação (EAp) afeta os índices de análise de crescimento do milho. Os melhores valores para os índices foram obtidos quando a aplicação foi realizada aos 36 e 43 dias após o plantio. Aos 120 dias de avaliação obteve-se os maiores valores para TCR, RMF e TCA, aos 90 dias de avaliação obteve-se a maior TCC.

\section{AGRADECIMENTOS}

Os autores agradecem ao Ministério da Ciência, Tecnologia, Inovações e Comunicações (MCTIC), à Fundação de Amparo à Pesquisa do Estado de Goiás (FAPEG), ao Conselho Nacional de Desenvolvimento Científico e Tecnológico (CNPq) e ao Instituto Federal Goiano (IF Goiano) pelo auxílio financeiro ao presente projeto de pesquisa.

\section{REFERÊNCIAS}

ALVAREZ, C. A.; STAPE, J. L.; SENTELHAS, P. C.; MORAES GOIÇALVES, J. L; SPAROVEK, G. Köppen's climate classification map for Brazil. Meteorologische Zeitschrift, Berlim, v. 22, n. 6, p. 711728, 2013. DOI: https://doi.org/10.1127/09412948/2013/0507
ALVAREZ, R. C. F.; CRUSCIOL, C. A. C.; NASCENTE, A. S. Análise de crescimento e produtividade de cultivares de arroz de terras altas dos tipos tradicional, intermediário e moderno. Pesquisa Agropecuária Tropical, Goiânia, v. 42, n. 4, p. 397-406, 2012. DOI: http://dx.doi.org/10.1590/S1983-40632012000400008

ANDRADE, A. C.; FONSECA, D. M. da; LOPES, R. dos S.; NASCIMENTO JÚNIOR, D. do; CECON, P. R.; QUEIROZ, D. S.; PEREIRA, D. H.; REIS, S. T. Análise de crescimento do capim-elefante 'Napier' adubado e irrigado. Ciência e Agrotecnologia, Lavras, v. 29, n. 2, p. 415-423, 2005. DOI: http://dx.doi.org/10.1590/S141370542005000200020

ANDRADE, C. A. B.; SCAPIN, C. A.; LUCCA, A.; BRACCINI, A. L.; MARTORELLI, D. T. Produtividade, crescimento e partição de matéria seca em duas cultivares de feijão. Acta Scientiarum. Agronomy, Maringá, v. 31, n. $4, \quad$ p. 683-688, 2009. DOI: http://dx.doi.org/10.4025/actasciagron.v31i4.6397

BENINCASA, M. M. P. Análise de crescimento de plantas: noções básicas. Jaboticabal: FUNEP, 2003. 42 p.

CONAB_Companhia Nacional de Abastecimento. Acompanhamento da safra brasileira de grãos, safra 2014/15, v. 2, n. 12. Brasília: CONAB, p. 1-134, 2015.

COSTA, C. C.; OliveIRA, C. D. de; SIlVA, C. J. da; TIMOSSI, P. C.; LEITE, I. C. Crescimento, 
produtividade e qualidade de raízes de rabanete cultivadas sob diferentes fontes e doses de adubos orgânicos. Horticultura Brasileira, Brasília, v. 24, n. 1, p. 118-122, 2006. DOI: http://dx.doi.org/10.1590/S010205362006000100024

DONAGEMMA, G. K.; CAMPOS, D. V. B.; CALDERANO, S. B.; TEIXEIRA, W. G.; VIANA, J. H. M. Manual de métodos de análises químicas de solo. 2 . ed. Rio de Janeiro: Embrapa Solos, 2011.230p.

FALQUETO, A. R.; CASSOL, D.; JÚNIOR MAGALHÃES, A. M.; OLIVEIRA, A. C.; BACARIN, M. A. Crescimento e partição de assimilados em cultivaresde arroz diferindo no potencial de produtividadede grãos. Bragantia, Campinas, v. 68, n. 3, p. 563-571, 2009. DOI: http://dx.doi.org/10.1590/S0006-87052009000300002

GUIMARÃES, C. M.; STONE, L. F.; NEVES, P. de C. F. Eficiência produtiva de cultivares de arroz com divergência fenotípica. Revista Brasileira de Engenharia Agrícola e Ambiental, Campina Grande, v. 12, n. 5, p. 465-470, 2008. DOI: http://dx.doi.org/10.1590/S1415-43662008000500004

JÚNIOR ORIOLI, V.; PRADO, R. M.; LEONEL, C. L.; CAZETTA, D. A.; SILVEIRA, C. M.; QUEIROZ, R. J. B.; BASTOS, J. C. H. A. G. Modos de aplicação de zinco na nutrição e na produção de massa seca de plantas de trigo. Revista de la ciencia del suelo y nutrición vegetal, Temuco, v. 8, n. 1, p. 28-36, 2008. DOI: http://dx.doi.org/10.4067/S0718-27912008000100003.

LACERDA, C. F.; CARVALHO, C. M.; VIEIRA, M. R.; NOBRE, J. G. A.; NEVES, A. L. R.; RODRIGUES, C. F. Análise de crescimento de milho e feijão sob diferentes condições de sombreamento. Revista Brasileira de Ciências Agrárias, Recife, v. 5, n. 1, p. 18-24, 2010. DOI: http://dx.doi.org/10.5039/agraria.v5i1a485

LIMA, J. L.; PEIXOTO, C. P.; LEDO, C. A. S. Índices fisiológicos e crescimento inicial de mamoeiro (caricapapaya 1.) em casa de vegetação. Ciência e agrotecnologia, Lavras, v. 31, n. 5, p. 1358-1363, 2007. DOI: $\quad$ http://dx.doi.org/10.1590/S141370542007000500013

LOPES, J. P.; MACHADO, E. C.; DEUDER, R.; MACHADO, R. S. Análise de crescimento e trocas gasosas na cultura de milho em plantio direto e convencional. Bragantia, Campinas, v. 68, n. 4, p. 839848, 2009. http://dx.doi.org/10.1590/S000687052009000400003.

LOPES, W. A. R; NEGREIROS, M. Z; DOMBROSKI, J. L. D; RODRIGUES, G. S. O; SOARES, A. M; ARAÚJO, A. P. Análise do crescimento de tomate 'SM-16' cultivado sob diferentes coberturas de solo. Horticultura Brasileira, Brasília, v. 29, n. 4, p. 554-561, 2011. DOI: http://dx.doi.org/10.1590/S0102-05362011000400019

MAGALHÃES, A. C. N. Análise quantitativa do crescimento. In: FERRI, M. G. 2 ed. Fisiologia Vegetal.
São Paulo: Editoras EPU; EDUSP, 1985. v. 1, p. 331350 .

MONTE, J. A.; PACHECO A. S.; CARVALHO D. F.; PIMENTEL, C. Influência do turno de rega no crescimento e produção do tomateiro no verão em Seropédica. Horticultura Brasileira, Brasília, v. 27, n. 2, p. 222-227, 2009. DOI: http://dx.doi.org/10.1590/S010205362009000200018

OLIVEIRA, R. A.; DAROS, E.; ZAMBON, J. L. C.; WEBER, H.; IDO, O. T.; ZUFFELLATO-RIBAS, K. C.; KOEHLER, H. S.; SILVA, D. K. T. Crescimento e desenvolvimento de três cultivares de cana-de-açúcar, em cana-planta, no estado do Paraná: Taxas de crescimento. Scientia Agrária, Curitiba, v. 6, n. 1-2, p. 85-89, 2005. DOI: http://dx.doi.org/10.5380/rsa.v6i1.4599

OLIVEIRA FILHO, A. F.; OLIVEIRA, F. A.; MEDEIROS, J. F.; MESQUITA, T. O.; ZONTA, E. Crescimento de cultivares de mamoneira sob doses de torta de mamona. Revista Verde de Agroecologia e Desenvolvimento Sustentável, Pombal, v. 5, n. 5, p. 18-24, 2010.

OLIVEIRA, D. E C.; RESENDE, O.; SMANIOTTO, T. A. S.; CAMPOS, R. C.; CHAVES, T. H. Cinética de secagem dos grãos de milho. Revista Brasileira de Milho e Sorgo, Sete Lagoas, v. 11, n. 2, p. 190-201, 2012. DOI: http://dx.doi.org/10.18512/19806477/rbms.v11n2p190-201

PRADO, R. M.; ROMUALDO, L. M.; ROZANE, D. E.; VIDAL, A. A.; MARCELO, A. V. Modos de aplicação de zinco na nutrição e na produção de matéria seca do milho brs 1001. Bioscience Journal, Uberlândia, v. 24, n. 1, p. 67-74, 2008.

SANTOS, V. M.; MELO, A. V.; SIEBENEICHLER, S. C.; CARSOSO, D. P.; BENÍCIO, L. P. F.; VARANDA, M. A. Physiological indices of seedlings of maize (Zea mays L.) under the action of biostimulants. Journal of Biotechnology and Biodiversity, Gurupi, v. 4, n. 3, p. 232-239, 2013.

SANTOS, H. G.; JACOMINE, P. K. T.; ANJOS, L. H. C.; OLIVEIRA, V. A.; LUMBRERAS, J. F.; COELHO, M. R.; ALMEIDA, J. A.; CUNHA, T. J. F.; OLIVEIRA, J. B. Sistema brasileiro de classificação de solos. Centro Nacional de Pesquisa de Solos. 4 ed. Brasília: Embrapa Produção de Informação; Rio de Janeiro: Embrapa Solos. 2014. $376 \mathrm{p}$

ZEPKA, A. P. S. Germinação, vigor de sementes e crescimento de plantas de trigo (Triticumaestivum L.) submetidas a diferentes doses de pendimethalin. 2007. 48f. Dissertação (Mestrado em Fisiologia Vegetal) Instituto de Biologia, Universidade Federal de Pelotas, Pelotas, 2007. 